\title{
The utility of tumor markers CA I25, CA I5-3, and CA 19-9 in assessing the response to therapy in pulmonary and pleural tuberculosis
}

\section{Canturk Tascı' \\ Sevket Ozkaya ${ }^{2}$ \\ Bikemgul Ozkara ${ }^{3}$ \\ Ergun Tozkoparan' \\ Metin Ozkan' \\ Nuri Karadurmus ${ }^{4}$ \\ Muhittin Serdar \\ Arzu Balkan' \\ Hayati Bilgic'}

'Gulhane Medical Faculty, Department of Pulmonary Diseases, Ankara;

${ }^{2} \mathrm{Dr}$ Suat Seren Education and

Research Hospital for Chest Diseases and Thoracic Surgery, Department of Pulmonary Medicine, Izmir; ${ }^{3}$ Department of Pulmonary Diseases, Military Hospital, Diyarbakir; ${ }^{4}$ Gulhane Medical Faculty, Department of Medical Oncology, Ankara; ${ }^{5}$ Gulhane Medical Faculty, Department of Biochemistry, Ankara, Turkey
Correspondence: Nuri Karadurmus Department of Internal Medicine, Gulhane Medical Faculty, Asagıeglence, Etlik, Ankara, Turkey

Tel +90 3123044000

Email drnkaradurmus@yahoo.com
This article was published in the following Dove Press journal:

OncoTargets and Therapy

21 November 2012

Number of times this article has been viewed

Aim: Both of the diagnosis and treatment evaluation are time-consuming conditions in patients with pulmonary and pleural tuberculosis. The aim of this study was to establish the validity of tumor markers CA 125, CA 15-3, and CA 19-9 in the diagnosis of pulmonary and pleural TB and to verify the success of the treatment protocol.

Patients and methods: The levels of tumor markers CA 125, CA 15-3, and CA 19-9 were measured before and after treatment in 67 TB patients, 54 of whom had pulmonary TB and 13 of whom had pleural TB. All values were compared with the results of a healthy control group of 44 subjects.

Results: CA 125 and CA 15-3 levels were significantly high when compared with those of the healthy control group and there was a significant decrease in both tumor marker levels after treatment in patients with pulmonary TB $(P<0.001$ and $P<0.004$, respectively). However, the difference found in CA 19-9 levels before and after treatment in patients with pulmonary TB was not statistically significant $(P<0.08)$. When the CA 125, CA 15-3, and CA 19-9 values of the pulmonary $\mathrm{TB}$ group before treatment were compared with that of the healthy control group, the results were statistically significant in all parameters except CA 19-9 $(P<0.001, P<0.001$, and $P<0.09$ for CA 125, CA 15-3, and CA 19-9, respectively). In the patients with pleural TB, CA 125 , CA 15-3, and CA 19-9 values did not change significantly after treatment.

Conclusion: The authors suggest that CA 125 and CA 15-3 tumor markers may be important for verification of the success of treatment protocol in pulmonary $\mathrm{TB}$, as the differences found for these tumor markers between the pre- and the posttreatment periods are statistically significant.

Keywords: TB, evaluation, treatment efficacy, tumor marker

\section{Introduction}

Pulmonary and pleural tuberculosis (TB) are both important public health issues today in Turkey and other parts of the world. ${ }^{1-3}$ Although the diagnosis of TB can be made easily, the standard isolation methods currently in use are quite slow - culture on conventional Löwenstein-Jensen medium requires 4-8 weeks. The spread of drugresistant TB has emphasized the need for rapid diagnosis. There are many methods to assess the activity of pulmonary TB. The most widely used technique for diagnosing active TB is sputum smear microscopy. ${ }^{4}$ However, in cases where a patient's sputum smear is negative, a decision for treatment can be made based on typical clinical symptoms, specific findings on a chest roentgenogram, a tuberculin skin test, and sometimes typical findings on high-resolution computed tomography. ${ }^{5}$ Recently, other methods such as measurement of serum neopterin and serum procalcitonin 
have been used in the diagnosis of TB. ${ }^{5-7}$ In pulmonary TB, efficacy of treatment is determined by an acid-resistant bacilli screening at the fifth month - but, that is a very longtime period for most patients. Some screenings performed during this period can verify the success of the treatment protocol (ie, can assess the response to the treatment). Pathological evaluation is preferred in the diagnosis of pleural TB. ${ }^{8}$ Adenosine deaminase measurement in pleural fluid is another diagnostic method used. ${ }^{9}$ There is also literature suggesting that certain tumor markers such as cancer antigen (CA) 125 are used both in diagnosis of TB and in evaluation of the efficacy of treatment. ${ }^{10}$ Although studies with these markers are generally focused on TB peritonitis, some investigators have studied these markers in patients with pulmonary and pleural TB. CA 125 and CA 15-3 are the markers mainly studied. Very few studies of these markers have included CA 19-9. ${ }^{1-17}$ The aim of this study was to investigate the importance of measuring these tumor markers before and after treatment of pulmonary and pleural TB to assess the disease activity and to monitor response to therapy.

\section{Material and methods}

Sixty-seven patients with pulmonary $(n=54)$ and pleural $(n=13)$ TB were enrolled in this prospective study over approximately a 2-year period (March 2005 to April 2007). Local ethics committee approval was obtained for the study, and informed consent was obtained from all study participants. The TB patients did not have any disease other than TB. Serum samples were obtained from the patients with pulmonary or pleural TB and were analyzed by bacteriological, pathological, or radiological methods to measure CA 125, CA 15-3, and CA 19-9. This procedure was repeated after a 6-month anti-TB treatment (isoniazid, rifampicin, ethambutol, and pyrazinamide for the first 2 months; then isoniazid and rifampicin for the remaining 4 months). Because the study was performed in a military hospital, all patients were male (mean age, $25 \pm 4.1$ years). The authors recruited 44 healthy male subjects (mean age, $29 \pm 6.7$ years) as the control group. Samples were obtained only once from the control group.

In 35 of the patients with pulmonary TB, sputum smear and culture results were positive, whereas 19 of the patients with pulmonary TB had negative sputum smear but positive culture results. Diagnoses of pleural TB were made based on cytological analysis of pleural fluid, increased pleural fluid adenosine deaminase levels, or histological examination of pleural biopsy specimens in twelve patients. A pleurectomy was needed in only one patient for diagnosis and treatment. The 54 patients with pulmonary TB were grouped according to the degree of sputum smear positivity as 1,2,3 and 4 positivity. The scoring for degree of acid-resistant bacilli positivity can be summarized as follows: 1 if there is one to two bacilli in 100 areas, 2 if there is one to nine bacilli in 10 areas, 3 if there is one to nine bacilli in each area, and 4 if there is more than nine bacilli in each area. The results for the TB patients were compared with the results of the healthy control group, and the results obtained before and after treatment were compared.

Obtained serums were stored at $-80 \mathrm{C}^{\circ}$ and were analyzed simultaneously. CA 125, CA 15-3, and CA 19-9 levels were measured using the electrochemiluminescence immunoassay technique and an Analytics E170 (Elecsys module) immunoassay analyzer (Roche Diagnostics $\mathrm{GmbH}$, Mannheim, Germany).

Statistical analyses were made using statistical software (SPSS programme 15.0; SPSS Inc, Chicago, IL). Descriptive statistics were shown as mean plus or minus standard deviation. For the statistical assessment of pre- and posttreatment results, paired sample $t$-test and Mann-Whitney $\mathrm{U}$ test were used. Correlations were determined by using Pearson and Spearmen tests. $P$-values $<0.05$ were accepted as statistically significant.

\section{Results}

As mentioned earlier, the study involved 54 patients with pulmonary TB, 13 patients with pleural TB, and 44 healthy volunteers (control group) as study groups. Sputum smear results of the 54 patients with pulmonary TB (data not shown) were as follows: four positive acid-fast bacilli in five patients $(9.2 \%)$, three positive in 16 patients $(33.7 \%)$, two positive in seven patients $(7.7 \%)$, and one positive in seven patients (7.7\%); sputum smears (in three samples) failed to demonstrate acid-fast bacilli in 19 patients $(28.4 \%)$, but culture results were positive. Pretreatment tumor markers of the patients with pulmonary $(n=54)$ and pleural TB $(n=13)$ were compared with the results of the healthy control group (Tables 1 and 2). A statistically significant difference was found in the pulmonary TB patients for CA 125 and CA 15-3 levels but not for CA 19-9 levels (Table 1). In the patients with pleural TB, the difference was not statistically significant when compared with the healthy control group (Table 2). 
Table I The levels of tumor markers in patients with pulmonary tuberculosis before treatment and in the healthy control group

\begin{tabular}{|c|c|c|c|}
\hline Marker & $\begin{array}{l}\text { Level in patients } \\
(n=54)^{a}\end{array}$ & $\begin{array}{l}\text { Level in control } \\
\text { group }(n=44)^{a}\end{array}$ & $P$-value \\
\hline CA I $25(\mathrm{U} / \mathrm{mL})$ & $40.94 \pm 34.54$ & $29.4 I \pm I I .5 I$ & $0.001 *$ \\
\hline CA I5-3 (U/mL) & $30.97 \pm 21.49$ & $22.83 \pm 13.47$ & $0.001 *$ \\
\hline CA I9-9 (U/mL) & $10.40 \pm 13.89$ & $10.01 \pm 9.49$ & 0.09 \\
\hline
\end{tabular}

Notes: avalues presented as mean plus or minus standard deviation; *statistically significant $(P<0.05)$.

Mean CA 125, CA 15-3, and CA 19-9 values before and after treatment in the pulmonary TB patients (Table 3) were as follows: CA 125, $40.94 \pm 34.54 \mathrm{U} / \mathrm{mL}$ (pretreatment) versus $26.33 \pm 15.46 \mathrm{U} / \mathrm{mL}$ (posttreatment) $(P<0.001)$ (Figure 1A); CA 15-3, $30.97 \pm 21.49 \mathrm{U} / \mathrm{mL}$ (pretreatment) versus $26.83 \pm 15.47 \mathrm{U} / \mathrm{mL}$ (posttreatment) $(P<0.004)$ (Figure 1B); and CA 19-9, $10.40 \pm 13.89 \mathrm{U} / \mathrm{mL}$ (pretreatment) versus $11.16 \pm 15.66 \mathrm{U} / \mathrm{mL}$ (posttreatment) $(P=0.08)$. In the patients with pleural TB there was no statistically significant difference found between the values (Table 4): CA 125, $41.82 \pm 29.23 \mathrm{U} / \mathrm{mL}$ (pretreatment) versus $28.96 \pm 10.61 \mathrm{U} / \mathrm{mL}$ (posttreatment) $(P=0.06) ; \mathrm{CA} 15-3,31.92 \pm 21.13 \mathrm{U} / \mathrm{mL}$ (pretreatment) versus $29.38 \pm 23.61 \mathrm{U} / \mathrm{mL}$ (posttreatment) $(P=0.20)$; and CA 19-9, $5.29 \pm 2.98 \mathrm{U} / \mathrm{mL}$ (pretreatment) versus $5.16 \pm 2.84 \mathrm{U} / \mathrm{mL}$ (posttreatment) $(P=0.18)$.

Higher serum CA 125 levels were obtained from the patients with a higher degree of sputum smear positivity $(r=0.341, P=0.012)$. This significant correlation was not found for the other tumor markers (CA 15-3: $r=0.032$, $P=0.817$; CA 19-9: $r=-0087, P=0.532$ ).

Another interesting finding for CA 125 was that if the CA 125 value was higher than the reference value $(35 \mathrm{U} / \mathrm{mL}$ ) at the moment of diagnosis, a significant decrease was observed after treatment in both pulmonary and pleural TB patients $(P<0.001)$. However, if the CA 125 value was not high before the treatment, this reduction was not significant (Figure 2).

Table 2 The levels of tumor markers in patients with pleural tuberculosis before treatment and in the healthy control group

\begin{tabular}{|c|c|c|c|}
\hline Marker & $\begin{array}{l}\text { Level in patients } \\
(n=13)^{a}\end{array}$ & $\begin{array}{l}\text { Level in control } \\
\text { group }(n=44)^{a}\end{array}$ & $P$-value \\
\hline CA I 25 (U/mL) & $41.82 \pm 29.23$ & $29.4 I \pm \mid I .5 I$ & 0.06 \\
\hline CA I5-3 (U/mL) & $31.92 \pm 21.13$ & $22.83 \pm 13.47$ & 0.06 \\
\hline CA I9-9 (U/mL) & $5.29 \pm 2.98$ & $10.01 \pm 9.49$ & 0.21 \\
\hline
\end{tabular}

Note: ${ }^{2}$ alues presented as mean plus or minus standard deviation.
Table 3 The pre- and posttreatment levels of tumor markers in patients with pulmonary tuberculosis

\begin{tabular}{|c|c|c|c|}
\hline Marker & $\begin{array}{l}\text { Pretreatment } \\
\text { level }^{\mathrm{a}}\end{array}$ & $\begin{array}{l}\text { Posttreatment } \\
\text { level }^{\mathrm{a}}\end{array}$ & $P$-value \\
\hline CA I25 (U/mL) & $40.94 \pm 34.54$ & $26.33 \pm 15.46$ & $0.00 I^{*}$ \\
\hline CA I5-3 (U/mL) & $30.97 \pm 21.49$ & $26.83 \pm 15.47$ & $0.004^{*}$ \\
\hline CA I9-9 (U/mL) & $10.40 \pm 13.89$ & $11.16 \pm 15.66$ & 0.08 \\
\hline
\end{tabular}

Notes: aValues presented as mean plus or minus standard deviation; *statistically significant $(P<0.05)$.

\section{Discussion}

CA 125 is a protein found on the surface of many ovarian cancer cells. It also can be found in other cancers and on a limited scale in normal tissue. ${ }^{18}$ Most often, the CA 125 test is used in diagnosing ovarian cancer and to check how well treatment for ovarian cancer is working or whether the ovarian cancer has relapsed. In some nonmalignant diseases (chronic renal failure, some autoimmune diseases, granulomatous liver diseases, pancreatitis, and pulmonary TB) elevated values can be detected. ${ }^{14}$

Some recent studies have reported increased serum CA 125 values found in peritoneal and pleural TB patients. ${ }^{19,20}$ Ozsahin et $\mathrm{al}^{10}$ showed that CA 125 measurement may be useful in the diagnosis of TB and in differentiation of active and inactive disease. ${ }^{10}$ Some articles have claimed that CA 125 can be used to follow up the efficacy of treatment. The measurement was performed before treatment and after 4,6 , and 36 months of treatment. When the cutoff value was taken as $31 \mathrm{U} / \mathrm{L}$, sensitivity and specificity were high $(97 \%$ and $100 \%$, respectively). The decrease was found to be statistically significant during this period. ${ }^{21}$

The present authors determined that normal CA 125 levels increase in pulmonary and pleural TB. This increase was positively correlated with the degree of smear positivity in pulmonary TB. In other words, a patient with a degree of sputum smear positivity of 4 had a higher serum CA 125 level than a patient with a negative smear. A significant decrease to the normal level was obtained with a 6-month anti-TB treatment (Figure 1). This decrease in CA 125 levels was obvious if the preliminary value was higher than the cutoff value of $35 \mathrm{U} / \mathrm{mL}$ (Figure 2). Therefore, the present authors claim that CA 125 can be used in the monitoring of treatment response in pulmonary TB patients. Fortún et $\mathrm{al}^{22}$ reported that CA 125 values increase in patients with pulmonary TB and decrease to normal values during treatment. According to reported articles, the CA 125 level evaluated in patients with a negative sputum acid-fast bacillus stain. ${ }^{22}$ Huang et $\mathrm{al}^{23}$ suggested that CA 125 serum levels - in 


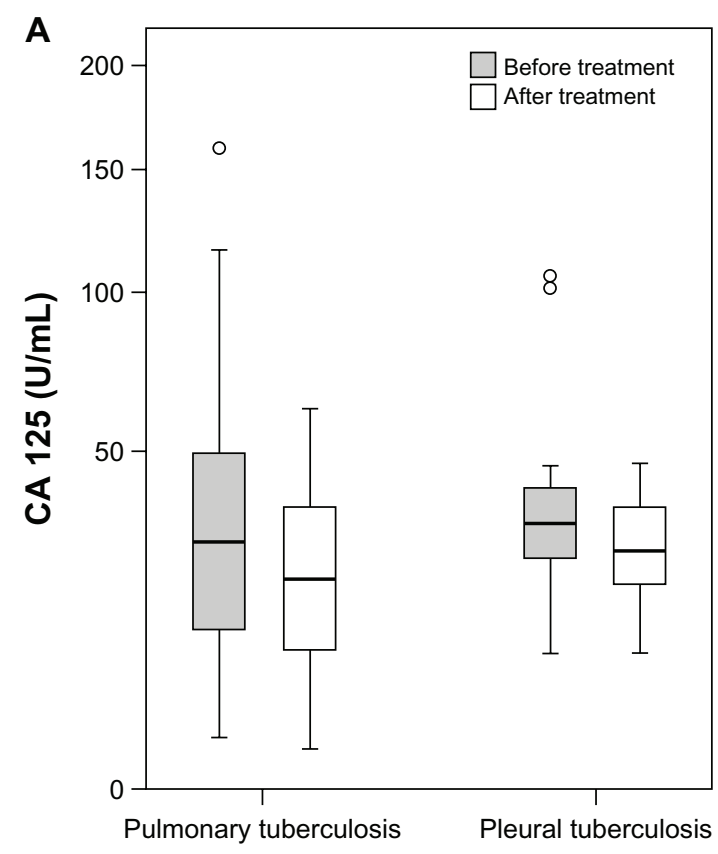

Figure I CA I25 (A) and CA I5-3 (B) values in pulmonary and pleural tuberculosis (TB).

combination with clinical responses, chest radiography, and sputum examinations - can offer improved monitoring of therapeutic responses in anti-TB treatment. ${ }^{23}$

CA 15-3 is a normal product of breast cells; it is produced by a gene that is often overexpressed in cancerous breast tumors, leading to an increased production of CA 15-3. ${ }^{15}$ However, CA 15-3 may also be elevated in some nonmalignant diseases such as chronic hepatitis, cirrhosis, sarcoidosis, TB, and systemic lupus erythematosus. ${ }^{15,16,24,25}$ A value greater than $25 \mathrm{U} / \mathrm{mL}$ is considered high.

Colomer et $\mathrm{al}^{15}$ determined an increase in CA 15-3 values in approximately $10 \%$ of patients with active TB. ${ }^{15}$ However, there are not enough data that demonstrate the role of CA 15-3 in evaluating the response to anti-TB treatment. The present study is, to the best of the authors' knowledge, the first study to establish the relationship between CA 15-3 and treatment response.

CA 19-9 is a tumor-associated marker. It is synthesized by normal human pancreatic and bile duct cells, as well

Table 4 The pre- and posttreatment levels of tumor markers in patients with pleural tuberculosis

\begin{tabular}{lccc}
\hline Marker & $\begin{array}{l}\text { Pretreatment }_{\text {level }^{\text {a }}} \\
\text { CA I25 (U/mL) }\end{array}$ & $\begin{array}{l}\text { Posttreatment } \\
\text { level }^{\mathbf{a}}\end{array}$ & P-value \\
CA 15-3 (U/mL) & $31.92 \pm 29.23$ & $28.96 \pm 10.61$ & 0.06 \\
CA 19-9 $(\mathrm{U} / \mathrm{mL})$ & $5.29 \pm 2.98$ & $29.38 \pm 23.61$ & 0.20 \\
\hline
\end{tabular}

Note: ${ }^{\vee}$ Values presented as mean plus or minus standard deviation.

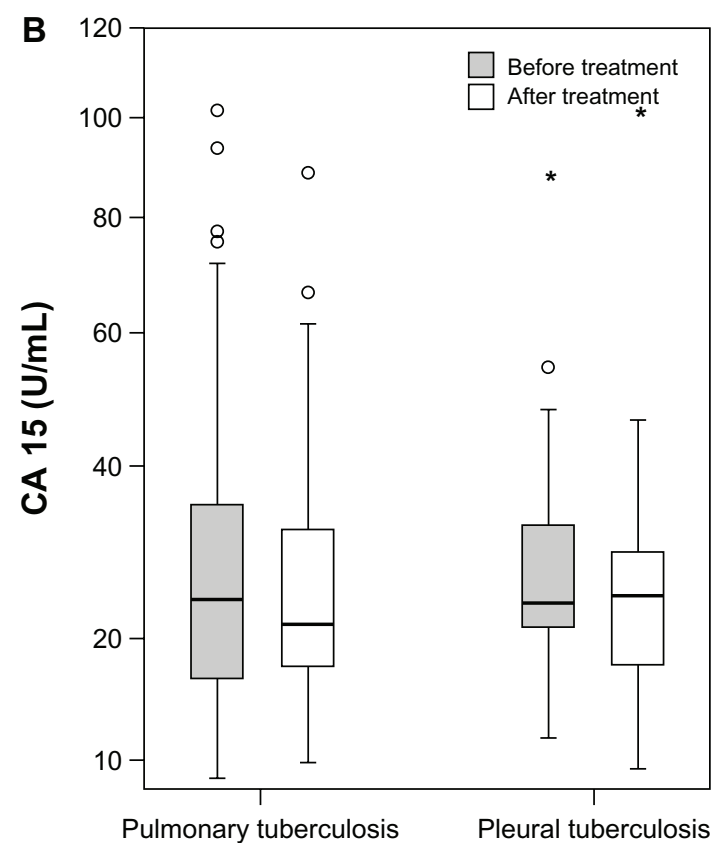

as by gastric, colonic, endometrial, salivary, and bronchial epithelia. ${ }^{12,13,17}$ Immunohistochemically, CA 19-9 is expressed in mucous cells of the bronchial gland and the surface of the bronchiolar epithelium cells in benign pulmonary disease. ${ }^{12}$ Usually it can be detected in malignant diseases but high levels are rarely found in benign liver and gallbladder diseases. ${ }^{17}$

Takayama et a ${ }^{12}$ measured serum CA 19-9 levels of 156 patients with benign pulmonary diseases. In 13 patients with healed pulmonary TB, the percentage of the patients with positive serum CA $19-9$ was $61.5 \%$. In the present study there was no significant correlation found between the disease activity and the response to treatment.

As already mentioned, there were 13 patients with pleural TB included in the present study. No significant increase was found in any parameter for these patients. If the patients were evaluated individually, both CA 125 and CA 15-3 levels were found to have decreased at the end of the treatment. However, it is possible that the number of patients in the study was not large enough for a statistical evaluation.

Although the routine use of tumor markers in all patients with TB cannot be approved, tumor markers can be useful in verification of the success of treatment protocol (ie, tumor markers can be useful in assessment of the response to treatment).

\section{Conclusion}

The CA 125 and CA 15-3 tumor markers correlate well with the disease activity in pulmonary TB. These two tumor 

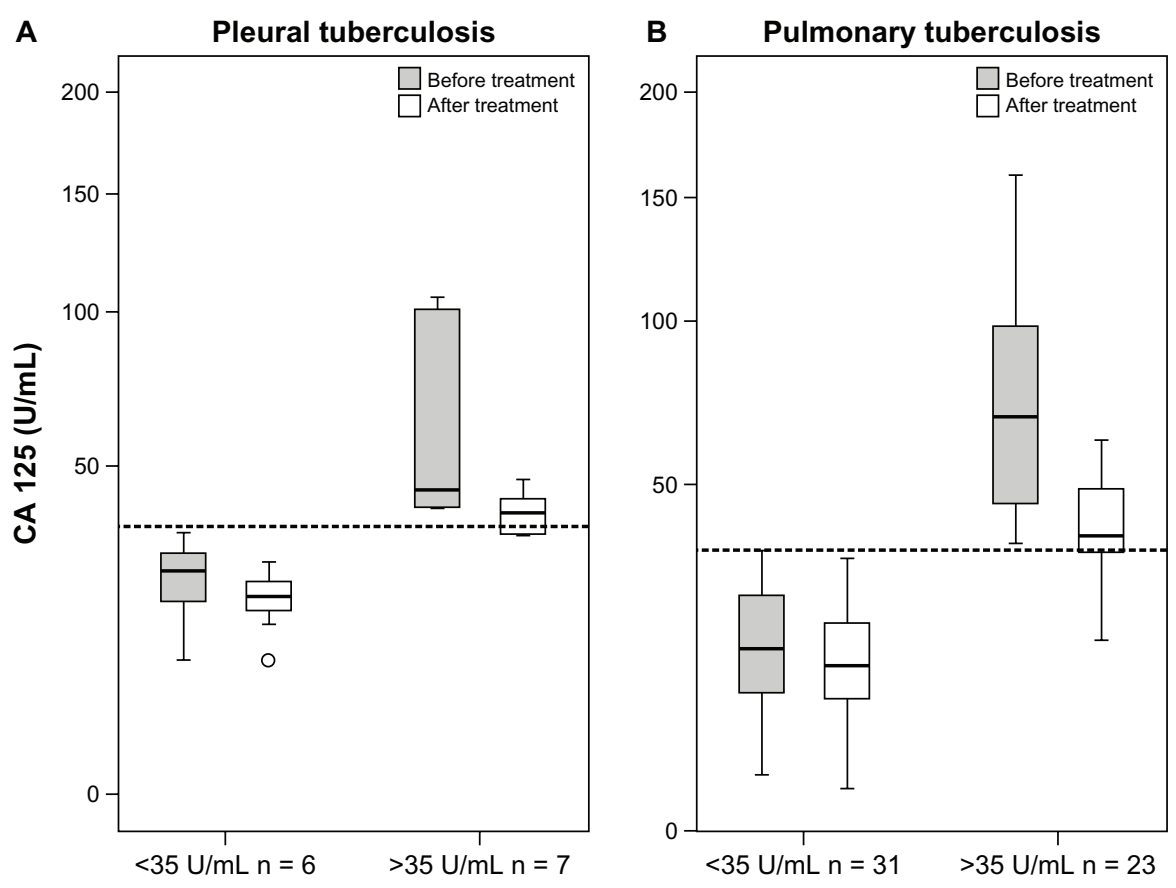

Figure 2 CA 125 in (A) pulmonary and (B) pleural tuberculosis (TB) when the cutoff value is taken as $35 \mathrm{U} / \mathrm{mL}$. Note: The decrease was significant when the initial values were higher than the cutoff value.

markers may be used to assess the response to treatment. The authors conclude that benign pulmonary diseases such as pulmonary TB affect serum tumor markers. In this study the authors suggest that determination of CA 125 and CA 15-3 levels in patients with pulmonary TB may be useful both in the diagnosis of illness and as a measure to provide verification of the efficacy of treatment protocol (ie, assess the response to treatment).

\section{Disclosure}

The authors report no conflicts of interest in this work.

\section{References}

1. Tanrikulu AC, Acemoglu H, Palanci Y, Dagli CE. Tuberculosis in Turkey: high altitude and other socio-economic risk factors. Public Health. 2008;122(6):613-619.

2. Sevim T, Ataç G, Güngör G, et al. Treatment outcome of relapse and defaulter pulmonary tuberculosis patients. Int J Tuberc Lung Dis. 2002; 6(4):320-325.

3. Aktoğu S, Yorgancioglu A, Cirak K, Köse T, Dereli SM. Clinical spectrum of pulmonary and pleural tuberculosis: a report of 5,480 cases. Eur Respir J. 1996;9(10):2031-2035.

4. Kilicaslan Z, Kiyan E, Kucuk C, et al. Risk of active tuberculosis in adult household contacts of smear-positive pulmonary tuberculosis cases. Int J Tuberc Lung Dis. 2009;13(1):93-98.

5. Ors F, Deniz O, Bozlar U, et al. High-resolution CT findings in patients with pulmonary tuberculosis: correlation with the degree of smear positivity. J Thorac Imaging. 2007;22(2):154-159.

6. Güler M, Hüddam D, Unsal E, et al. The role of serum neopterin level in the evaluation of activation and response to treatment in the patients with pulmonary tuberculosis. Tuberk Toraks. 2006;54(4):330-335. Turkish.
7. Balbay ÖA, Türker G, Çalışır HC, Şengül A. As a diagnostic markers of active pulmonary tuberculosis procalcitonin place. Solunum. 2000;11:299-303. Turkish.

8. Chikumi H, Shimizu E. Diagnostic significance of pleural effusion and pleural biopsy. Nihon Naika Gakkai Zasshi. 2000;89(5):874-883. Japanese.

9. Chen ML, Yu WC, Lam CW, Au KM, Kong FY, Chan AY. Diagnostic value of pleural fluid adenosine deaminase activity in tuberculous pleurisy. Clin Chim Acta. 2004;341(1-2):101-107.

10. Ozsahin SL, Turgut B, Nur N, Dogan OT, Erselcan T, Berk S. Validity of the CA125 level in the differential diagnosis of pulmonary tuberculosis. Jpn J Infect Dis. 2008;61(1):68-69.

11. Wani AM, Akhtar M. CA-125: a marker for diagnosis and follow-up of pleuroperitoneal and lymph node tuberculosis. Ann Saudi Med. 2008;28(2):142-143.

12. Takayama S, Kataoka N, Usui Y, et al. CA 19-9 in patients with benign pulmonary diseases. Nihon Kyobu Shikkan Gakkai Zasshi. 1990;28(10):1326-1331. Japanese.

13. Komiya T, Matsushima T, Kimura M, Adachi M. A case of endobronchial tuberculosis with high serum CA19-9 and SLX level. Kekkaku. 1994;69(10):615-619. Japanese.

14. Yilmaz A, Ece F, Bayramgürler B, Akkaya E, Baran R. The value of Ca 125 in the evaluation of tuberculosis activity. Respir Med. 2001; 95(8):666-669.

15. Colomer R, Ruibal A, Genollá J, et al. Circulating CA 15-13 levels in the postsurgical follow-up of breast cancer patients and in non-malignant diseases. Breast Cancer Res Treat. 1989;13(2):123-133.

16. Ustün H, Borazan A, Bilgiçli N, Yılmaz A. Diagnostic value of tumoural markers in pleural effusions. Int J Clin Pract. 2004;58(1): $22-25$.

17. Ishiura Y, Fujimura M, Minami S, et al. Increased CA19-9 level in serum and bronchoalveolar lavage fluid from a patient with pulmonary tuberculosis. Nihon Kyobu Shikkan Gakkai Zasshi. 1996;34(4): 477-481. Japanese.

18. Simsek H, Savas MC, Kadayifci A, Tatar G. Elevated serum CA 125 concentration in patients with tuberculous peritonitis: a case-control study. Am J Gastroenterol. 1997;92(7):1174-1176. 
19. Mas MR, Cömert B, Sağlamkaya U, et al. CA-125: a new marker for diagnosis and follow-up of patients with tuberculous peritonitis. Dig Liver Dis. 2000;32(7):595-597.

20. Uzunköy A, Nazlıül Y. Peritoneal tuberculosis: review. Turkiye Klinikleri J Med Sci. 2006;26:404-408.

21. Yilmaz A, Ece F, Bayramgürler B, Akkaya E, Baran R. The value of CA 125 in the evaluation of tuberculosis activity. Respir Med. 2001;95(8):666-669.

22. Fortún J, Martín-Dávila P, Méndez R, et al. CA-125: a useful marker to distinguish pulmonary tuberculosis from other pulmonary infections. Open Respir Med J. 2009;3:123-127.
23. Huang WC, Tseng CW, Chang KM, Hsu JY, Chen JH, Shen GH. Usefulness of tumor marker CA-125 serum levels for the follow-up of therapeutic responses in tuberculosis patients with and without serositis. Jpn J Infect Dis. 2011;64(5):367-372.

24. Ghayumi SM, Mehrabi S, Doroudchi M, Ghaderi A. Diagnostic value of tumor markers for differentiating malignant and benign pleural effusions of Iranian patients. Pathol Oncol Res. 2005;11(4):236-241.

25. Schöniger-Hekele M, Müller C. The combined elevation of tumor markers CA 19-9 and CA 125 in liver disease patients is highly specific for severe liver fibrosis. Dig Dis Sci. 2006;51(2):338-345.

\section{Publish your work in this journal}

OncoTargets and Therapy is an international, peer-reviewed, open access journal focusing on the pathological basis of all cancers, potential targets for therapy and treatment protocols employed to improve the management of cancer patients. The journal also focuses on the impact of management programs and new therapeutic agents and protocols on

\section{Dovepress}

patient perspectives such as quality of life, adherence and satisfaction. The manuscript management system is completely online and includes a very quick and fair peer-review system, which is all easy to use. Visit http://www.dovepress.com/testimonials.php to read real quotes from published authors. 